The Last Word

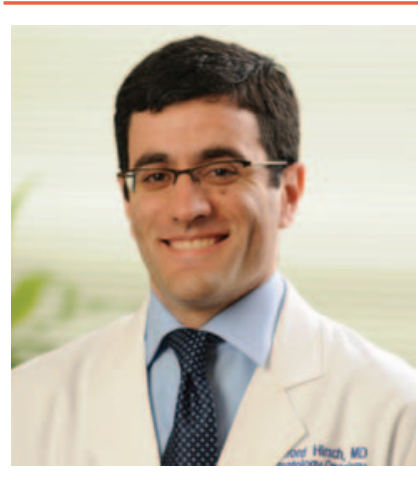

Bradford R. Hirsch, MD

Bradford R. Hirsch, MD, is an Assistant Professor of Medicine in the Department of Medical Oncology, Duke University School of Medicine and the Director of Clinical Informatics and Learning Laboratories at the Duke Center for Learning Health Care. He works to help reform the evidence development system in the United States in order to develop a learning health care system.
The ideas and viewpoints expressed in this editorial are those of the author and do not necessarily represent any policy, position, or program of NCCN.

\section{Incorporating the Patient's Voice in the Continuum of Care}

\author{
Bradford R. Hirsch, MD, and Amy P. Abernethy, MD
}

Clinicians may not want to admit it, but we aren't always the best source of information regarding a cancer patient's symptoms and experience with their disease. When it comes to the interpretation of laboratory results and imaging abnormalities, our input is a critical piece of the puzzle. But when it comes to reporting a patient's perception of their experience and the impact of interventions on it, we often misinterpret the responses. ${ }^{1}$ Although many feel that this has limited impact on the care being delivered, a growing body of evidence and opinion argues otherwise. The gold standard for documenting a patient's experience can only be the patient's own voice, so it is imperative that they be queried directly. ${ }^{2}$ In a setting of breakneck technologic advances, the pressing question is not whether the patient's voice can be heard, but how to most efficiently and reliably record it.

\section{The Oncologist's Lens}

Routine clinical practice is very different from the structured framework of a clinical trial, in which patient-reported outcomes (PROs) are increasingly required data elements. Even in those settings, the PROs that are collected are not used to inform the care of a particular trial patient. Incorporating PROs into the routine clinic visit is logical — we treat a patient, not a tumor — but the logistics of efficiently and reliably obtaining PROs and being able to integrate them with other data at the point of care remains a stumbling block. Also at issue is timing. A given patient is not seen on a daily basis. It may be weeks or months between visits, and much can happen in the intervening time. Add to that our increasingly hectic clinic schedules, the intermittent nature of many symptoms, and the variable levels of trusting communication between clinicians, patients, and their caregivers, and the result is often a struggle to optimize the quality of life for our patients with cancer.

It cannot be assumed that a patient will have the opportunity to bring up a symptom's time course and trajectory of intensity during a visit, or that our inquiries will capture the patient's experience. We may rarely ask about a specific concern that is pressing to a given patient and a patient may not be comfortable bringing up sensitive issues, such as sexual distress. ${ }^{3}$ A patient may not understand the significance of a given symptom or it might not be particularly pressing on the day of an appointment, much as an unusual sound from your engine never seems to be heard when you take your car to the mechanic. These issues within health care are not reflected in the data generated today and are not voiced in the satisfaction scores that patients report, but we all know the frustration that patients feel. We also feel powerless at times to do anything about it.

\section{The Patient's Lens}

"Today is my clinic appointment. I have noticed a painful numbness in my hands that made it difficult to get dressed today. It is not a big thing, but a bother nonetheless. I planned to ask about it, but the clinic is running late today and my doctor will have to rush through my appointment. I'll just keep that annoyance to myself instead of wasting her time and ask at my next appointment. If things get worse over the next month, there will be plenty of time to discuss it." 
Even if the neuropathy is discovered during a thorough review of systems by the clinic staff, the trajectory may not be apparent to the clinician and the patient has already decided to minimize it. The appointment concludes, but the information exchange is incomplete. Instead of discussing a dose reduction, potential therapeutic interventions, and warnings about what to do if the pain gets worse, the symptom will progress for another month. As a clinician, we have all asked a patient, "Why didn't you tell me about that earlier?"

\section{Ensuring the Patient's Voice is Heard-Efficiently and With Maximal Impact}

We've all experienced the impact of having increasingly less time to spend with patients to understand their experiences and educate them about their disease. At the same time, we feel increasing pressure to improve the quality of our care and prevent complications and hospitalizations. There is also increasing pressure to trim staff and control costs. How do we reconcile these clearly opposing trends? One answer is to incorporate the patient's voice directly into care in ways that can be documented, analyzed, and acted on.

Numerous methods are available for collecting a patient's thoughts. A paper and pencil survey filled out in the waiting room is the most basic entry point, but it is inadequate for billing, must be scanned into the electronic chart, and cannot be tracked or analyzed over time. It is not efficient or effective, and, if the patient perceives that the information is not being used, it will yield less and less information at subsequent visits.

Novel mechanisms to capture the patient voice are readily available, and have been shown to be both feasible and reliable. ${ }^{4}$ The introduction of electronic health records, increasingly ubiquitous smart phones, mobile health (mHealth) technologies (eg, iPads, Kindles), and other Internet-enabled platforms (eg, home computers, digital televisions) have opened a new window to the patient's experience. Interfaces have been developed at the Center for Learning Health Care at Duke University, West Clinic (a large community oncology practice based in Memphis), and at other institutions, that allow patients and caregivers to provide longitudinal input about their symptoms and concerns. ${ }^{5}$ Data can be collected anywhere and the collection process is defined by the needs of the clinic and patients. Using a secure server, the information is uploaded, analyzed, and stored, enabling innumerable uses for the data. A printout is generated for the clinical team before the patient's name is even called in the waiting room that provides a snapshot of the symptoms, including trends and areas in need of prompt attention. Extensive patient surveys and stakeholder interviews demonstrate that patients value the approach, it helps them remember and report important concerns, and they are willing to answer up to 100 questions at each clinical encounter, provided that clinicians look at the report and that it is used to trigger meaningful actions. ${ }^{4}$

At the Duke Cancer Institute, studies are underway to make this basic functionality even more robust. Patient-reported data will "belong to patients" and follow them across health systems and traditional information boundaries. Responses will prompt the delivery of tailored education across platforms, such as the release of an educational video to a patient's iPad that outlines the management of nausea. Data obtained from patients are made available to the physician at the next clinic appointment or, if a specific threshold is reached or pressing concern is broached, interventions such as a call from a clinic nurse will be proactively triggered, instead of waiting for a distressed patient call when the issue has become emergent.

New information technologies often prompt a visceral response from clinicians and administrators because of a fear of additional workload, decreased productivity,

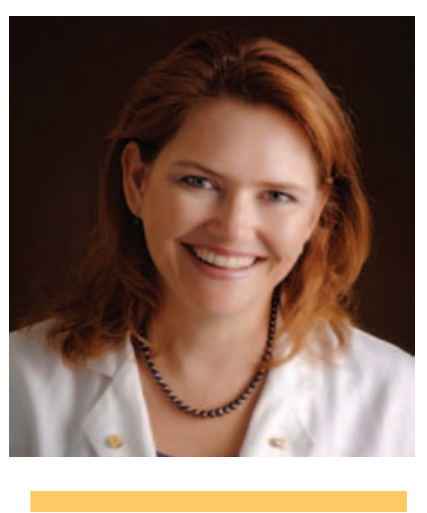

Amy P. Abernethy, MD

Amy P. Abernethy, MD, directs the Center for Learning Healthcare (CLHC) in the Duke Clinical Research Institute. She also directs the Duke Cancer Care Research Program (DCCRP). As Director of the CLHC, Dr. Abernethy spearheads the organization to develop and test practical solutions to facilitate learning health care delivery systems and evidence-based, patient-centered care. Dr. Abernethy is an internationally recognized expert in health services research and delivery in patient-centered cancer care, symptom management, and palliative care. 
increased risk, and limited impact on relevant outcomes. Discussions of PRO platforms are often no different, because they represent yet another data source to monitor and worry about. However, mHealth technologies, such as the PRO system, have fundamental differences that lead to great promise. If PRO capture can be integrated successfully into the clinical workflow, it replaces and augments actions that are already occurring. All clinics collect a review of systems, all clinicians educate their patients, and all systems deal with the complications of therapies. Dictation time is shortened and the solutions ensure complete review of systems documentation to facilitate coding and billing, with $17 \%$ improvement in efficiency shown. Through augmenting education, monitoring patients for symptom control, and proactively addressing concerns outside of traditional clinical settings, entirely new care processes are possible. However, they are possible, despite requiring limited, if any, input from clinicians outside of the initial design process.

The value of routinely incorporating PRO data collection goes beyond the obvious positive impact on the clinical encounter. Patient-reported data connects physicians, nurses, social workers, psychosocial care providers, palliative care providers, and chaplains through providing information on a patient's pain, quality of life, and distress. It can be linked to administrative databases, electronic health records, clinical research datasets, and basic science research to enhance quality monitoring, comparative effectiveness research, clinical research, and discovery science. PRO data can be linked to biorepositories to contribute key phenotypic information. The authors have recently shown the use of routinely collected PROs to tailor patient education, screen patients for clinical trials, monitor cancer care quality at point-ofcare, and assess the effectiveness of care delivery models in their clinics. ${ }^{6,7}$

The themes of patient-centered care, personalized medicine, and learning health systems are all gaining traction nationally. ${ }^{8,9}$ Without the correct systems in place to support these activities, they will remain a conversation as opposed to a reality. Routinely collected patient-reported information supports clinical care and ensures that the patient voice is at the center of evolving health care models.

\section{References}

1. Pakhomov SV, Jacobsen SJ, Chute CG, Roger VL. Agreement between patient-reported symptoms and their documentation in the medical record. Am J Manag Care 2008;14:530-539.

2. Basch $E$. The missing voice of patients in drug-safety reporting. N Engl J Med 2010;362:865-869.

3. Dupont A, Wheeler J, Herndon JE II, et al. Use of tablet personal computers for sensitive patientreported information. J Support Oncol 2009;7:91-97.

4. Abernethy AP, Herndon JE II, Wheeler JL, et al. Feasibility and acceptability to patients of a longitudinal system for evaluating cancer-related symptoms and quality of life: pilot study of an e/ Tablet data-collection system in academic oncology. J Pain Symptom Manage 2009;37:1027-1038.

5. Abernethy AP, Herndon JE II, Wheeler JL, et al. Improving health care efficiency and quality using tablet personal computers to collect research-quality, patient-reported data. Health Serv Res 2008;43:1975-1991.

6. Abernethy AP, Ahmad A, Zafar SY, et al. Electronic patient-reported data capture as a foundation of rapid learning cancer care. Med Care 2010;48(6 Suppl):S32-38.

7. Bull J, Zafar SY, Wheeler JL, et al. Establishing a regional, multisite database for quality improvement and service planning in community-based palliative care and hospice. J Palliat Med 2010;13:10131020.

8. Greene SM, Reid RJ, Larson EB. Implementing the learning health system: from concept to action. Ann Intern Med 2012;157:207-210.

9. Bardes CL. Defining “patient-centered medicine”. N Engl J Med 2012;366:782-783. 